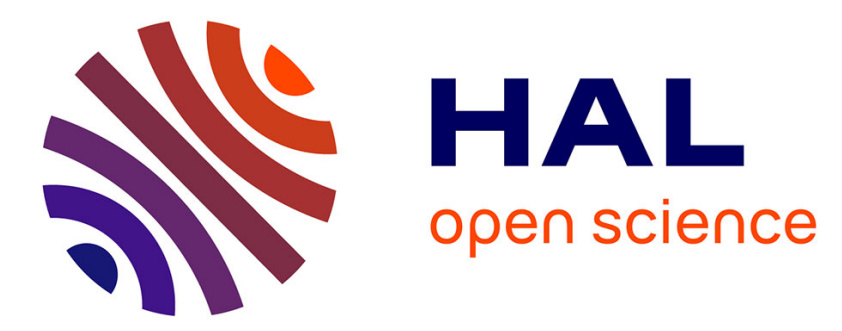

\title{
Investigating the origin of acoustic attenuation in liquid foams
}

Juliette Pierre, Camille Gaulon, Caroline Derec, Florence Elias, Valentin Leroy

\section{To cite this version:}

Juliette Pierre, Camille Gaulon, Caroline Derec, Florence Elias, Valentin Leroy. Investigating the origin of acoustic attenuation in liquid foams. European Physical Journal E: Soft matter and biological physics, 2017, 40 (8), pp.73. 10.1140/epje/i2017-11562-0 . hal-02008640v2

\section{HAL Id: hal-02008640 \\ https://hal.science/hal-02008640v2}

Submitted on 20 Apr 2023

HAL is a multi-disciplinary open access archive for the deposit and dissemination of scientific research documents, whether they are published or not. The documents may come from teaching and research institutions in France or abroad, or from public or private research centers.
L'archive ouverte pluridisciplinaire HAL, est destinée au dépôt et à la diffusion de documents scientifiques de niveau recherche, publiés ou non, émanant des établissements d'enseignement et de recherche français ou étrangers, des laboratoires publics ou privés. 


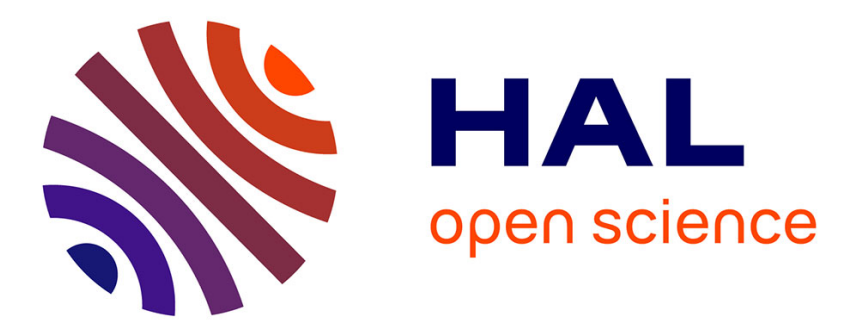

\section{Investigating the origin of acoustic attenuation in liquid foams}

Juliette Pierre, Camille Gaulon, Caroline Derec, Florence Elias, Valentin Leroy

\section{To cite this version:}

Juliette Pierre, Camille Gaulon, Caroline Derec, Florence Elias, Valentin Leroy. Investigating the origin of acoustic attenuation in liquid foams. 2017. hal-01536661

\section{HAL Id: hal-01536661 \\ https://hal.science/hal-01536661}

Preprint submitted on 12 Jun 2017

HAL is a multi-disciplinary open access archive for the deposit and dissemination of scientific research documents, whether they are published or not. The documents may come from teaching and research institutions in France or abroad, or from public or private research centers.
L'archive ouverte pluridisciplinaire HAL, est destinée au dépôt et à la diffusion de documents scientifiques de niveau recherche, publiés ou non, émanant des établissements d'enseignement et de recherche français ou étrangers, des laboratoires publics ou privés. 


\title{
Investigating the origin of acoustic attenuation in liquid foams
}

\author{
Juliette Pierre $^{1 \mathrm{a}}$, Camille Gaulon ${ }^{2}$, Caroline Derec $^{2}$, Florence Elias $^{3}$, and Valentin Leroy ${ }^{2 \mathrm{~b}}$ \\ 1 Sorbonne Universités, UPMC Univ Paris 06, CNRS (UMR 7190), Institut Jean Le Rond d'Alembert, Paris, France \\ 2 Laboratoire Matière et Systèmes Complexes, Université Paris-Diderot, Sorbonne Paris Cité, CNRS (UMR 7057), Paris, France \\ 3 Sorbonne Universités, UPMC Univ Paris 06, CNRS (UMR 7057), laboratoire Matière et Systèmes Complexes, Paris, France
}

the date of receipt and acceptance should be inserted later

\begin{abstract}
Liquid foams are known to be highly efficient to absorb acoustic waves but the origin of the sound dissipation remains unknown. In this paper, we present low frequency $(0.5-4 \mathrm{kHz})$ experimental results measured with an impedance tube and we confront the recorded attenuations to a simple model that considers the foam as a concentrate bubbly liquid. In order to identify the influence of the different parameters constituting the foams we probe samples with different gases, and various liquid fractions and bubble size distributions. We demonstrate that the intrinsic acoustic attenuation in liquid foam is due to both thermal and viscous losses. The physical mechanism of the viscous term is not elucidated but the microscopic effective viscosity evidenced here can be described by a phenomenological law scaling with the bubble size and the gas density. In our experimental configuration a third dissipation term occurs. It comes from viscous friction on the wall of the impedance tube and it is well described by Kirchhoff law considering the macroscopic effective viscosity classically measured in rheology experiments.
\end{abstract}

PACS. 47.57.Bc Complex fluids and colloidal systems: Foams and emulsions - 47.35.Rs Sound waves in fluid dynamics

\section{Introduction}

Liquid foams are complex materials with a high fraction of gas bubbles dispersed in a liquid matrix and stabilised by surfactants. Contrary to fibrous materials and solid foams, liquid foams have received less attention for their acoustical properties. Recently, progress has been made on the understanding of how sound propagates in such media. For instance, the existence of two regimes of propagation was evidenced: so-called Wood regime at low frequencies, and so-called Kann one at higher frequencies [1]. In the Wood regime [2], the whole structure of the foam moves under the effect of the pressure wave, and the effective velocity is given by the usual mixture law, typically of the order of $30 \mathrm{~m} / \mathrm{s}$ for a foam with a $\Phi=10 \%$ liquid volume fraction. In the regime proposed by Kann [3], only the films move and the effective velocity is higher, typically of the order of $200 \mathrm{~m} / \mathrm{s}$.

Most of the experimental studies on the acoustics of liquid foams also report a quite strong level of attenuation, even capable of significantly reducing the amplitude of blast waves for instance [4] . However, interestingly, the origin of this attenuation is not clear. There is still a debate on whether dissipation is mainly viscous or thermal. Some authors claim that thermal losses dominate, and can explain their observation $[5,6]$. Their argument is based

\footnotetext{
a email: juliette.pierre@upmc.fr

b email: valentin.leroy@univ-paris-diderot.fr
}

on a scaling law. If one notes $k$ the complex wavenumber, it can be shown that the reduced thermal attenuation $\tilde{\alpha}=\operatorname{Im}(k) / \operatorname{Re}(k)$ is expected to scale like $\omega R^{2} / D_{\text {th }}$, where $\omega$ is the angular frequency, $D_{\text {th }}$ the thermal diffusivity of the gas, and $R$ the typical radius of the bubbles in the foam. The problem is that, if the scaling law in $\omega R^{2}$ was indeed observed in several experimental studies $[5,6]$, other authors pointed out that the order of magnitude of the thermal attenuation was not enough to explain the experimental results $[7,8]$. Even more intriguing, experiments with different types of gas showed that the $\tilde{\alpha} \sim 1 / D_{\text {th }}$ scaling law was not respected [9].

In this article, we propose to bring fresh experimental observations to this debate on the origin of acoustic attenuation in liquid foams. Section 2 presents our experimental setup, which enabled us to measure the acoustic attenuation between 0.4 and $4 \mathrm{kHz}$ for liquid foams with various bubble size distributions, liquid fractions and gas content. In section 3, we expose the acoustic attenuation predicted by a simple model that considers the foam as a concentrated bubbly liquid. The limit of this approach, which does not account for the complex structure of the foam, will be discussed. In section 4, we present our experimental results and we analyse the dependence of the attenuation with the different parameters (frequency, gas nature, liquid fraction, bubbles size). We show that two sources of attenuation are well identified: viscous losses on the walls and thermal losses in the bubbles. A third 
contribution, whose mechanism is not clear yet, is necessary to explain the measurements. In section 5 , we finally conclude by giving a phenomenological law for this contribution.

\section{Materials and methods}

Liquid foams of well determined structure and composition were made (section 2.1) and their acoustical properties were measured with an impedance tube (section 2.2).

\subsection{Sample preparation}

Foam generation. Liquid foams were generated by using the "double-syringe technique" [10], which consists in filling a syringe with the desired amount of foaming solution and gas, then connecting to another syringe and pushing back and forth the pistons to obtain an homogeneous foam. With this technique, providing that the solution has a good "foamability", a large range of liquid volume fractions $(\Phi)$ can be obtained, just by adjusting the proportion of liquid and gas in the syringe. In our case, we typically explored $\Phi$ varying from 3 to $25 \%$.

Foaming solution. We worked with a foaming solution for which a negligible interfacial elasticity was expected: SDS (Sodium Dodecyl Sulfate) at $10 \mathrm{~g} / \mathrm{L}$ in millipore water. The density was the same as that of water: $\rho_{\ell}=$ $10^{3} \mathrm{~kg} / \mathrm{m}^{3}$.

Gas composition. In order to evidence the influence of the gas properties on the sound attenuation, we used different types of gas. The easiest gas to use was air. We also used helium, from an helium balloon cylinder. We obtained another type of gas by using vapor of perfluorohexane. Perfluorohexane $\left(\mathrm{C}_{6} \mathrm{~F}_{14}\right)$ is liquid at normal conditions of temperature and pressure, but its vapor pressure is high $\left(25 \mathrm{kPa}\right.$ at $\left.20^{\circ} \mathrm{C}\right)$, so a significant amount of vapor is obtained at atmospheric pressure. We used a closed bottle with a small amount of liquid perfluorohexane to obtain air saturated with vapor of perfluorohexane (molar fraction of 0.2 ). A fourth type of thermal property was obtained by using half air from the room and half gas from the bottle to fill the syringe. Table 1 summarises the properties of the four types of gas we used. Note, that, for clarity, specific symbols are used in the whole article for each gas (see Table 1).

Bubble size measurements. The bubble size distribution was obtained by the "bubble raft" technique [11], which consists in spreading a small amount of the sample on the surface of a foaming solution bath, and then imaging the $2 \mathrm{D}$ structure obtained (see inset of figure 1). Size distributions were found to be well fitted by lognormal laws, with
Table 1. Gas composition and properties: ratio of heat capacities $\gamma$, thermal diffusivity $D_{\text {th }}$ and gas density $\rho_{g}$. For gas mixtures, molar fractions are given (figures in brackets). See Appendix A for details on how properties of gas mixture were estimated.

\begin{tabular}{|c|c|c|c|c|c|}
\hline & name & composition & $\gamma$ & $\begin{array}{c}D_{\text {th }} \\
\left(\mathrm{mm}^{2} / \mathrm{s}\right)\end{array}$ & $\begin{array}{c}\rho_{g} \\
\left(\mathrm{~kg} / \mathrm{m}^{3}\right)\end{array}$ \\
\hline$\triangle$ & air & pure air & 1.4 & 20 & 1.2 \\
\hline$\diamond$ & He & $\begin{array}{c}\text { helium (0.75), } \\
\text { air (0.25) }\end{array}$ & 1.57 & 128 & 0.42 \\
\hline$\square$ & mix & $\begin{array}{c}\mathrm{C}_{6} \mathrm{~F}_{14}(0.1), \\
\text { air (0.9) }\end{array}$ & 1.19 & 11.5 & 2.5 \\
\hline$\circ$ & $\mathrm{PFH}$ & $\begin{array}{c}\mathrm{C}_{6} \mathrm{~F}_{14}(0.2), \\
\text { air }(0.8)\end{array}$ & 1.12 & 7.5 & 3.8 \\
\hline
\end{tabular}

a median radius $R$ and a polydispersity index $\mathrm{PI}^{1}$. Smallest bubble sizes were obtained by using the foam sample right after their production $(R \simeq 20 \mu \mathrm{m})$. Larger bubbles were obtained by letting the foam age in the syringe before doing the acoustic measurements. The maximum size we could explore was $R \simeq 80 \mu \mathrm{m}$ because for samples with larger bubbles, the attenuation was found to be too high for a proper analysis of the signal. Figure 1 shows typical examples of cumulative distributions obtained for small, intermediate and large bubbles.

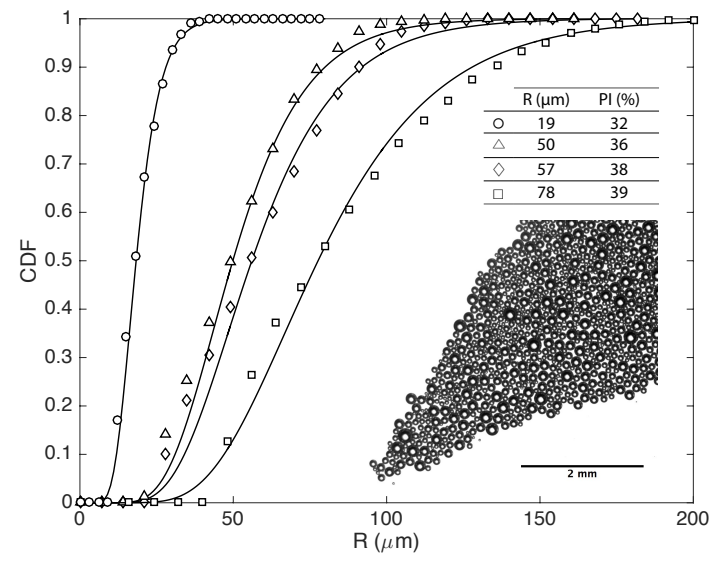

Fig. 1. Four examples of cumulative size distributions, as measured by analysis of $2 \mathrm{D}$ rafts, such as the one depicted in the inset (here for the distribution shown by triangles). Solid lines show the best-fitted lognormal laws, whose parameters (median radius $R$ and polydisperse index PI) are summarised in the inset table.

Summary of the samples. Figure 2 proposes a diagram to visualise the range of structure parameters we were able to explore. Note that, as a first approximation, we will consider that the size distribution is fully described

\footnotetext{
${ }^{1} \mathrm{PI}=\sqrt{\ln \left(1+\sigma^{2}\right)}$ where $\sigma$ is the normalised standard de-
} viation of the bubble size distribution 
by the median radius $R$, the polydispersity being similar for all the samples. The structure of the foam is thus given by $\Phi$, whose role was investigated over the $4-20 \%$ range, and $R$, which varied between 15 and $80 \mu \mathrm{m}$. Figure 2 also distinguishes the type of gas used for producing the foam (symbols). It appears that the whole range of $R$ is not covered by all the types of gas. This is because ageing of the foam strongly depends on the gas content. On one hand, foams with perfluorohexane (PFH and mix) will age slowly, giving us access to small bubbles: bubbles size initiates at $15 \mu \mathrm{m}$ and reaches $60 \mu \mathrm{m}$ in 2 hours. On the other hand, foams with air or helium contain larger bubbles: with air the initial bubble size is around $40 \mu \mathrm{m}$ and grows to $100 \mu \mathrm{m}$ in 5 minutes. As a consequence, investigating the role of the gas for foams of identical structure can only be done on a limited range of bubble sizes. In figure 2 , we highlight 4 samples whose median radii were close to $50 \mu \mathrm{m}$ and liquid volume fraction of the order of $10 \%$ (see Table 2). These samples will be used to explore the role of the gas on the acoustic attenuation.

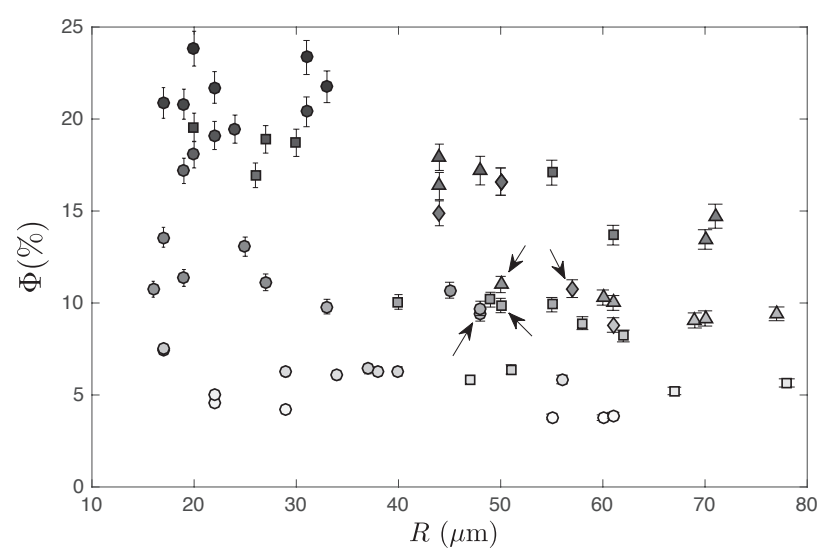

Fig. 2. Liquid volume fractions $(\Phi)$ and bubble median sizes $(R)$ for the 71 foam samples investigated. Symbols code for the type of gas (see Table 1), the grey level of the markers correspond to liquid fraction (the darker the symbols the wetter the foams) and arrows indicate the four selected samples with similar structure and different gas content (see Table 2).

Table 2. Type of gas, liquid fraction, median bubble radius and polydispersity index for the four foam samples highlighted in Fig. 2. Gas content is different but structure similar.

\begin{tabular}{|c|c|c|c|c|}
\hline & gas & $\Phi(\%)$ & $R(\mu \mathrm{m})$ & PI $(\%)$ \\
\hline$\triangle$ & air & 11 & 50 & 36 \\
$\bullet$ & $\mathrm{PFH}$ & 9.5 & 48 & 23 \\
$\square$ & $\operatorname{mix}$ & 10 & 50 & 30 \\
$\diamond$ & $\mathrm{He}$ & 11 & 57 & 38 \\
\hline
\end{tabular}

\subsection{Acoustic measurements}

The acoustical properties of the foams were measured with an impedance tube (B\&K, type 4206), schematised in figure $3 \mathrm{a}$. The principle is to measure the reflection coefficient $\mathcal{R}$ on a sample of given thickness $d$. The tube is held vertically, with the foam sample at the bottom, and the measurement is performed quickly enough (a few seconds) to prevent drainage and ageing of the foam. Typical result of a measurement is shown in Fig. 3b, in which the real and imaginary parts of $(1-\mathcal{R}) /(1+\mathcal{R})$ are plotted as function of the frequency. A series of peaks appear, which are related to resonances of the foam layer. Each peak can be analysed and we showed in a previous article [8] that the position of a peak depended on the sound velocity in the foam $(v)$, its width on the attenuation $(\alpha)$, and its height on the density of the sample $(\rho)$. Thus, by analysing the different peaks, we were able to determine $v, \alpha$, and $\rho$ as functions of frequency, for a frequency range of $0.4-4 \mathrm{kHz}$. Details on the analysis of the peaks can be found in Ref [8].
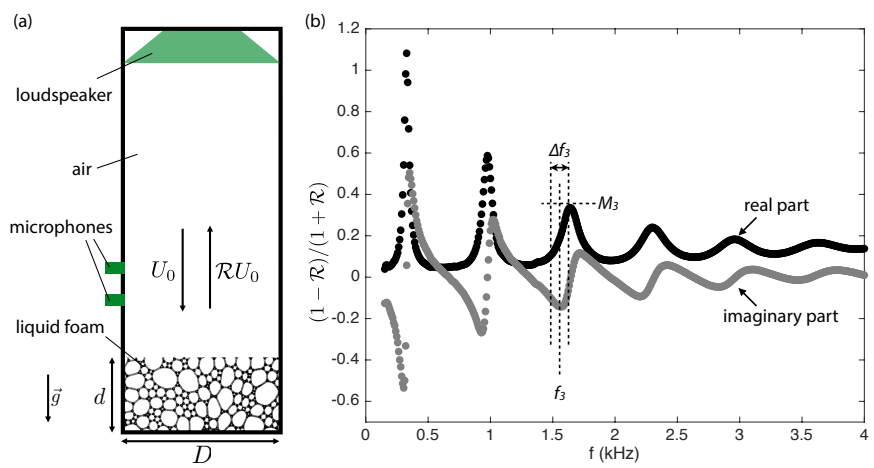

Fig. 3. Acoustic setup and typical acoustic measurement. (a) The impedance tube is held vertically and the bottom is filled with liquid foam on a thickness $d$. The reflection coefficient $\mathcal{R}$ of the foam sample is then determined by measuring the pressure field with two wall-mounted microphones. (b) Plotting $(1-\mathcal{R}) /(1+\mathcal{R})$ as a function of frequency shows a series of peaks, whose position, width and height allow us to determine the velocity, attenuation and density of the foam. Here the example is for a foam made of air saturated with $\mathrm{C}_{6} \mathrm{~F}_{14}$, with $\Phi \approx 10 \%$ and $R=48 \mu \mathrm{m}$.

To illustrate the kind of measurements we obtained, we show in figure 4 the measured density, sound velocity, and sound attenuation as functions of frequency, for the four foams selected in Table 2. Error bars come from the uncertainty on the foam thickness $d(d=2.5 \pm 0.1 \mathrm{~cm})$. Density and velocity are found to be almost independent on both frequency and gas content. On the other hand, the attenuation has a large frequency and gas dependence. The helium foam is the most attenuating, and $\mathrm{PFH}$ sample the least attenuating. In order to highlight the role played by the foam structure and the gas composition in the acoustic dissipation process, we now focus on the attenuation, first theoretically, and then experimentally, studying the samples presented in Fig. 2. 

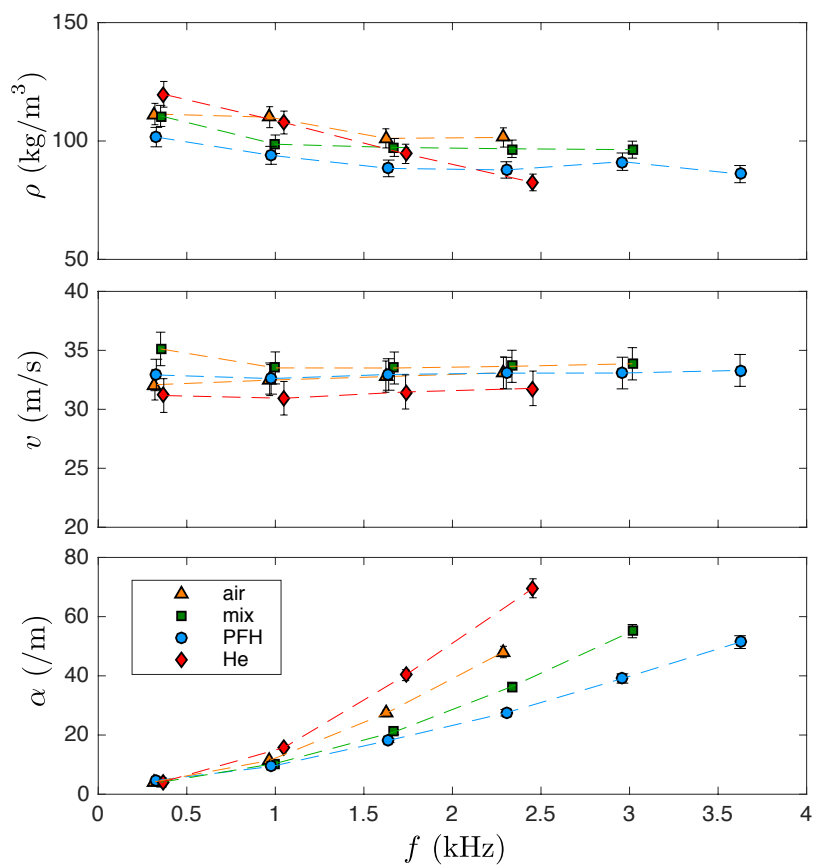

Fig. 4. From top to bottom, density $\rho$, velocity $v$ and attenuation $\alpha$ as functions of frequency for the four selected foams of Table 2, which have similar structures but different gas contents (color online).

\section{Theory}

In this section, we present theoretical results for the velocity and attenuation of sound in a liquid foam. We will consider propagation at the long wavelength limit, i.e. when the frequency is low enough for the wavelength to be much larger than the typical size of the heterogeneities in the medium. In this article, the largest bubble median radius we consider is of the order of $0.1 \mathrm{~mm}$, while the highest frequency is $4 \mathrm{kHz}$. At this frequency, the wavelength of sound is of 85 and $375 \mathrm{~mm}$ in air and water, respectively. Hence, we are in the proper conditions for modeling the foam as an effective medium.

We propose to first consider the liquid foam as a bubbly liquid (subsection 3.1), because the theory for acoustic propagation in those media, based on the physics of a single bubble oscillating in an infinite extent of liquid, is well developed. Note that this is also the approach chosen in most of the previous theoretical studies on the acoustics of liquid foams $[5,6,12]$. We will then discuss how this approach could be modified to account for the fact that, in foams, bubbles are beyond the close-packing configuration (subsection 3.2). This discussion will give us guidelines for the analysis of the experimental results.

\subsection{Acoustic propagation in a bubbly liquid}

We consider that the bubbly liquid has an effective density $\rho_{\text {eff }}$ and an effective compressibility $\chi_{\text {eff }}$, leading to an effective acoustic wavenumber $k$ :

$$
(k / \omega)^{2}=\rho_{\mathrm{eff}} \chi_{\mathrm{eff}},
$$

where $\omega$ is the angular frequency.

Let us assume that the effective density is given by a simple mixture law:

$$
\begin{aligned}
\rho_{\mathrm{eff}} & =\rho_{\ell} \Phi+\rho_{g}(1-\Phi), \\
& \simeq \rho_{\ell} \Phi, \quad \text { for } \Phi \gg 0.1 \%,
\end{aligned}
$$

where $\rho_{\ell}$ and $\rho_{g}$ are respectively the mass density of the liquid and the gas. Given the high density contrast between the gas and liquid, (2b) is a very good approximation for most liquid foams. Experimental evidence of large deviation from this mixture law was observed $[3,5,12,13]$ and recently explained [1], showing the existence of a resonance that depends on the size of the bubbles. However, given the typical bubble sizes and frequency range we consider here, resonant effects are not expected to be significant: for a median radius of $0.1 \mathrm{~mm}$, the resonance frequency is expected at $25 \mathrm{kHz}$, above our $4 \mathrm{kHz}$ maximal frequency.

As for the effective compressibility, it is defined by $\chi_{\text {eff }}=-(1 / V) \partial V / \partial P$, where $P$ is the pressure applied to volume $V$. If we consider that this volume contains bubbles of radius $R$ and a volume of liquid $V_{\ell}=\Phi V$, we obtain

$$
\chi_{\mathrm{eff}}=-3(1-\Phi) \frac{1}{R} \frac{\partial R}{\partial P}+\Phi \chi_{\ell},
$$

where $\chi_{\ell}=-\left(1 / V_{\ell}\right) \partial V_{\ell} / \partial P$ is the liquid compressibility. We see that the effective compressibility thus depends on the bubble dynamics at the considered frequency. Following Rayleigh-Plesset theory [14], we can consider three pressure terms that affect the oscillations of the bubble: a thermal term, an inertial one, and a viscous one. ${ }^{2}$ We then obtain, neglecting the compressibility of the liquid, the following expression for the effective wavenumber:

$$
\left(\frac{k}{\omega}\right)^{2}=\frac{\rho_{\ell} \phi(1-\phi)}{\kappa P_{0}-\frac{\omega^{2} \rho_{\ell} R_{0}^{2}}{3}-\frac{4 \mathrm{i} \eta \omega}{3}},
$$

where $\kappa$ is the complex polytropic exponent of the gas, $P_{0}$ the static pressure in the bubbles, and $\eta$ the viscosity of the liquid. Note that $k$ is now a complex number: the bubble's response is not necessarily in phase with the sound wave, which induces attenuation. This expression can be further simplified by noting that, for the range of sizes and frequencies investigated here, the inertial term is negligible. At the limit of low attenuation $(\operatorname{Im}(k) \ll$ $\operatorname{Re}(k)$ ), we thus obtain the following expressions for the effective velocity $(v=\omega / \operatorname{Re}(k))$ and reduced attenuation $(\tilde{\alpha}=\operatorname{Im}(k) / \operatorname{Re}(k))$ :

$$
\begin{aligned}
v & =\sqrt{\frac{\operatorname{Re}(\kappa) P_{0}}{\rho_{\ell} \Phi(1-\Phi)}}, \\
\tilde{\alpha} & =\tilde{\alpha}_{\mathrm{th}}+\tilde{\alpha}_{\mathrm{vi}} \\
& =\frac{-\operatorname{Im}(\kappa)}{2 \operatorname{Re}(\kappa)}+\frac{2 \eta \omega}{3 \operatorname{Re}(\kappa) P_{0}} .
\end{aligned}
$$

${ }^{2}$ Note that we neglect surface tension and radiative effects here. 
Equation (5) is usually known as Wood's law. It was checked to properly predict phase velocity in liquid foams [8], provided that the resonance could be neglected.

Equation (6) shows that the reduced attenuation is the sum of a thermal and a viscous term. For bubbly water $(\eta=1 \mathrm{mPa} . \mathrm{s})$, the latter term is of the order of $7 \times 10^{-7} \omega$. Thermal loss, on the other hand, depends on the imaginary part of the complex polytropic exponent, which depends on the ratio between the thermal length $\ell_{\mathrm{th}}=\sqrt{2 D_{\mathrm{th}} / \omega}$ and bubbles radius $R[14]$ :

$$
\kappa=\frac{\gamma}{1+3(\gamma-1) \frac{1-X \operatorname{cotan}(X)}{X^{2}}},
$$

where $X=(1+\mathrm{i}) R / \ell_{\text {th }}$ and $\gamma$ is the ratio of the specific heats. Eq. (7) predicts isothermal transformations of the gas $(\operatorname{Re}(\kappa) \simeq 1)$ at low frequencies $\left(\ell_{\mathrm{th}}>R\right)$, and adiabatic ones $(\operatorname{Re}(\kappa) \simeq \gamma)$ at high frequencies $\left(\ell_{\mathrm{th}}<R\right)$. For the low frequency regime, imaginary part of equation (7) can be approximated by

$$
\operatorname{Im}(\kappa) \simeq \frac{1-\gamma}{15 \gamma} \times \frac{R^{2} \omega}{D_{\mathrm{th}}},
$$

which gives the $\omega R^{2} / D_{\text {th }}$ scaling law discussed in the introduction. For $R=50 \mu \mathrm{m}$ air bubbles eq. (8) predicts a reduced attenuation of the order of $\tilde{\alpha}_{\text {th }} \simeq 10^{-6} \omega$.

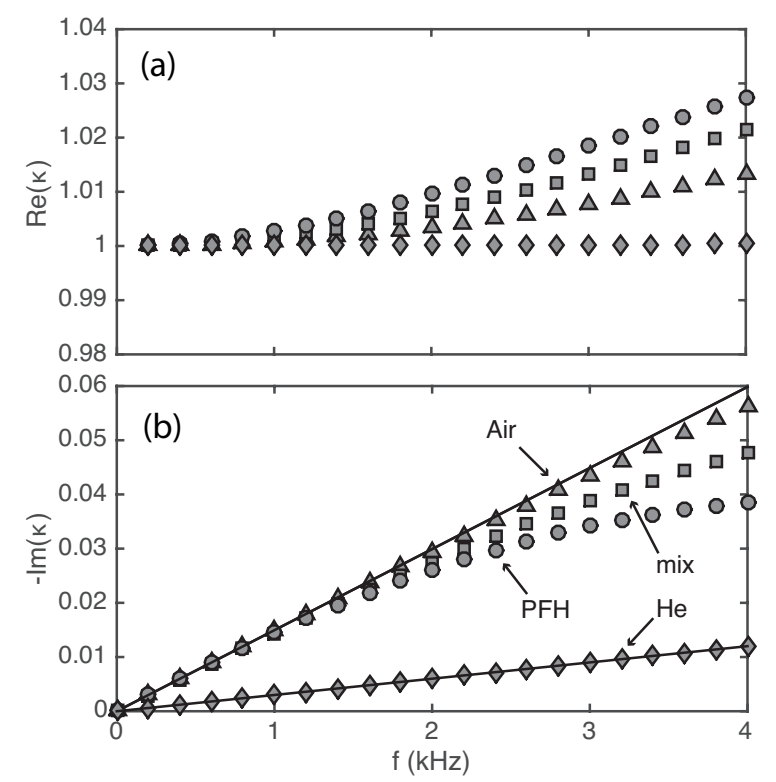

Fig. 5. Theoretical prediction of equation (7) for the real (a) and imaginary (b) part of the polytropic exponent as function of frequency, for the four types of gas considered in Table 1. The bubble size is taken at $R=50 \mu \mathrm{m}$. Symbols code for the type of gas reported in Table 1. Continuous lines show the low frequency approximation given by Eq. (8), for air and helium.

Fig. 5 shows predictions of Eq. (7) for the four gases considered in Table 1 , for a radius $R=50 \mu \mathrm{m}$. We see that thermal behaviour is expected to be almost isothermal in our experiments, with an imaginary part of $\kappa$ close to law (8) for the lowest frequencies, but with deviation at higher frequencies. We will see that this deviation is even more pronounced when polydispersity is taken into account (see Fig. 8).

\subsection{From bubbly liquid to liquid foam}

The structure of a liquid foam is different from that of the dilute bubbly liquid considered for obtaining equation (6): bubbles are not isolated, but in contact with each other. As a consequence, instead of being uniformly distributed around the bubbles, the liquid phase takes two forms: thin films and thicker liquid channels (see Fig. 6). Hence the question arises whether the thermal and viscous behaviours can be described by the same equations as for the dilute case.

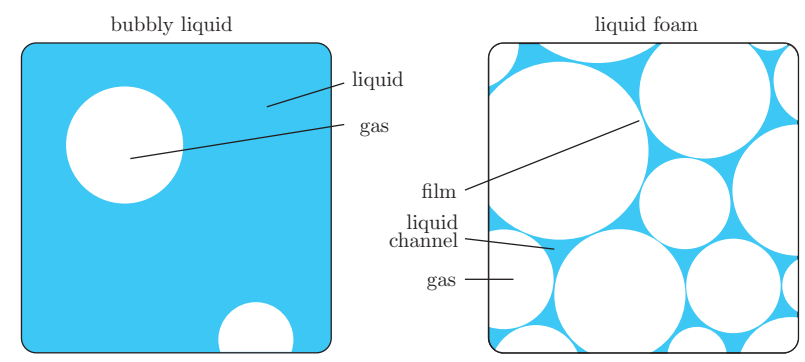

Fig. 6. In a bubbly liquid (left), gas bubbles are far from each other. In a foam (right), the structure is more complex because bubbles are in close contact: the liquid phase is distributed in channels and films. Note that bubbles are spherical in this scheme for simplicity. In reality, beyond the close packing limit $(\Phi<36 \%)$ sphericity is lost.

Thermal losses. To obtain equation (7), it is assumed that the liquid around the bubble remains at the same temperature during the oscillations. In a foam, the liquid content is much less than in a dilute bubbly liquid, and this assumption is questionable. Goldbfarb et al [7] considered a bubble surrounded by a liquid shell of finite size, adapted to the liquid volume fraction of the foam, and calculated the thermal behaviour of this system. With this description, it appears that there is enough liquid to maintain the condition of constant temperature. One can argue, however, that, in a foam, bubbles are not covered by an homogeneous layer of liquid: the liquid is distributed between thick liquid channels and thin films. As films are expected to be only a few tens of nanometers thick [15], thermal exchanges between two neighbouring bubbles might be significant. We show, in appendix $\mathrm{B}$, that the presence of thin films may indeed affect the validity of equation (7). However, as deviations are expected to be small for the wet foams we consider here, we shall assume in the following that thermal attenuation in a foam is the same as in a bubbly liquid. 
Viscous losses. The viscosity one needs to consider in equation (6) is not clear when the oscillating bubbles is surrounded by other bubbles. Let us denote by $\eta_{\text {bub }}$ this viscosity:

$$
\tilde{\alpha}_{\mathrm{vi}} \simeq \frac{2 \eta_{\mathrm{bub}} \omega}{3 P_{0}}
$$

(considering that $\operatorname{Re}(\kappa) \simeq 1$ ). We examine three possible choices for $\eta_{\text {bub }}$ and give the resulting expected scaling law in $\omega, R$ and $\Phi$ for $\tilde{\alpha}_{\mathrm{vi}}$ :

$-\eta_{\mathrm{bub}}=\eta_{\text {water }}$. If we assume that the viscous losses are the same as in the dilute bubbly liquid case, the viscosity is that of the foaming solution, i.e. close to the viscosity of water. In this case, we expect $\tilde{\alpha}_{\mathrm{vi}} \sim$ $\omega^{1} \Phi^{0} R^{0}$ (no dependence in $\Phi$ and $R$ )

$-\eta_{\text {bub }}=\eta_{\text {foam }}$. We can consider the effective viscosity of the foam itself. Rheology of liquid foams has been studied extensively. The following expression for the viscosity was shown to be in good agreement with measurements [16]:

$$
\eta_{\text {foam }}=A \sqrt{1+(B / R)^{2}} \omega^{-1 / 2},
$$

with $A=10.3 \mathrm{~Pa}_{\mathrm{s}} \mathrm{s}^{1 / 2}$ and $B=52 \mu \mathrm{m}$. The scaling law for the reduced viscous attenuation is then expected to be $\tilde{\alpha}_{\mathrm{vi}} \sim \omega^{1 / 2} \Phi^{0} R^{-1}$ for $R \ll 52 \mu \mathrm{m}$ and $\tilde{\alpha}_{\mathrm{vi}} \sim$ $\omega^{1 / 2} \Phi^{0} R^{0}$ for $R \gg 52 \mu \mathrm{m}$.

- $\eta_{\text {bub }}=\eta_{\text {channel }}$. Goldfarb and coworkers [7] proposed that when the bubble was oscillating, it displaced liquid in the channel network. Using a Darcy law for describing the flow, they obtained the following effective viscosity:

$$
\eta_{\text {channel }} \simeq \eta_{\text {water }}\left(1+\frac{71}{\Phi}\right)
$$

which would lead to scaling law $\tilde{\alpha}_{\mathrm{vi}} \sim \omega^{1} \Phi^{-1} R^{0}$.

In the next part, we will present our experimental results and compare them to Eq. (6) prediction.

\section{Experimental results}

In this section, we first present measurements of the sound velocity in our foam samples, which are found to be in agreement with Wood's law (section 4.1). We then focus on the reduced attenuation $\tilde{\alpha}$ as a function of frequency for four selected foam samples and show that thermal attenuation alone cannot explain the measurements (section 4.2). We also identify a third mechanism of dissipation, due to the friction losses on the wall of the tube (section 4.3). We then present our fitting procedure for determining the values of two parameters that pilot the attenuation: $\eta_{\text {bub }}$ and $\eta_{\text {wall }}$ (section 4.4). "Wall viscosity" $\eta_{\text {wall }}$ is found to be well described by the macroscopic viscosity of the foam $\left(\eta_{\text {foam }}\right)$. We finally discuss the dependence of "bubble viscosity" $\eta_{\text {bub }}$ on the structure and composition of the foam (section 4.5).

\subsection{Wood's law}

In figure 7 we plot, for each sample, the measured sound velocity as a function of the (acoustically measured) density. As already observed in previous studies [8], we see that the measurements are well described by the isothermal Wood's law. Note that, as the bubbly liquid approach predicts such an isothermal behaviour (see Fig. 5a), this observation supports our hypothesis that thermal losses are also well described by this approach.

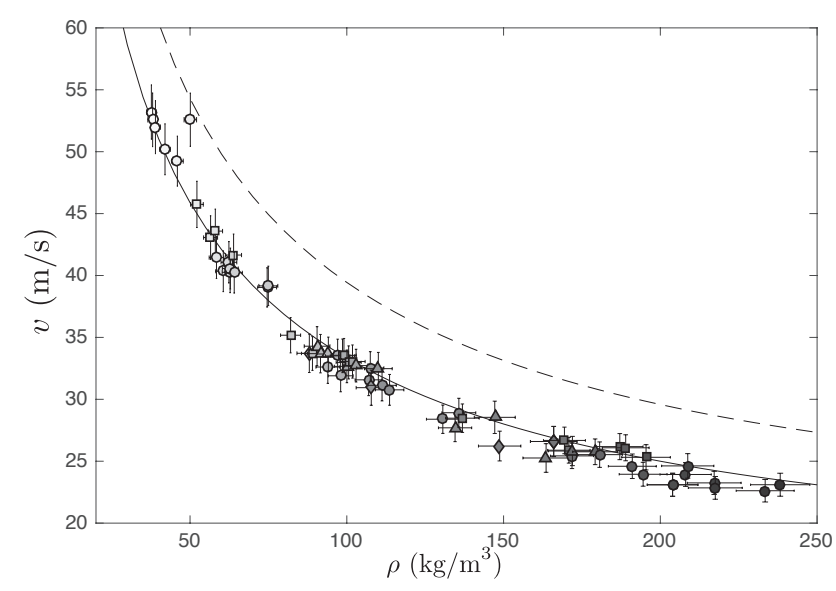

Fig. 7. Sound velocity as a function of the acoustic density at $1 \mathrm{kHz}$ for the 71 samples investigated. Symbols code for the type of gas of Table 1 . The grey level of the symbols correspond to liquid fraction: the darker the symbols the wetter the foams (same as fig. 2). Continuous line shows the isothermal Wood's law $(\operatorname{Re}(\kappa)=1)$, while the dashed one represents the adiabatic law for air $(\operatorname{Re}(\kappa)=1.4)$.

\subsection{Examples of measured reduced attenuation}

To illustrate the typical reduced attenuations measured in our experiments, we show the raw measurements obtained for the four samples selected in Table 2 (see symbols in top part of Fig. 8a). Based on the discussion in the theoretical section (see 3.2), we will assume that the thermal attenuation is well-described by the bubbly liquid approach. As a consequence, because the structure of the foams and their gas composition are known, we can fully calculate the expected behaviour of $\tilde{\alpha}_{\text {th }}$ as a function of frequency. We can even take the polydispersity into account, by integrating on the whole bubble size distribution. Figure 8a shows, in solid lines, the predicted thermal reduced attenuation. In line with the simplified monodisperse cases presented in Fig. 5, we see that thermal attenuation is expected to be higher for air foams, then for "mix" and "PFH" samples, and finally for helium foams. The maximum expected reduced attenuation is of the order of 0.04 , which is clearly insufficient to explain the measurements (symbols in Fig. 8a). Let us stress here that, even if deviation from the bubbly liquid approach appeared, because 
of the presence of thin films, one would expect a lower thermal attenuation (see Appendix B). Moreover, the attenuation is found to be maximal in the helium samples, in contradiction with what is expected for thermal losses. We thus conclude from figure 8 a that thermal losses alone cannot explain acoustic attenuation in liquid foams.

Another clear feature of figure $8 \mathrm{a}$ is the non-monotonic behaviour of $\tilde{\alpha}$, which is first decreasing with frequency, and then increasing. This behaviour is even clearer when one subtracts the theoretical thermal contribution from the measured reduced attenuation, as shown in Fig. 8b. We show in the next section that the low frequency part of the measured attenuation is due to viscous losses on the wall of the impedance tube.

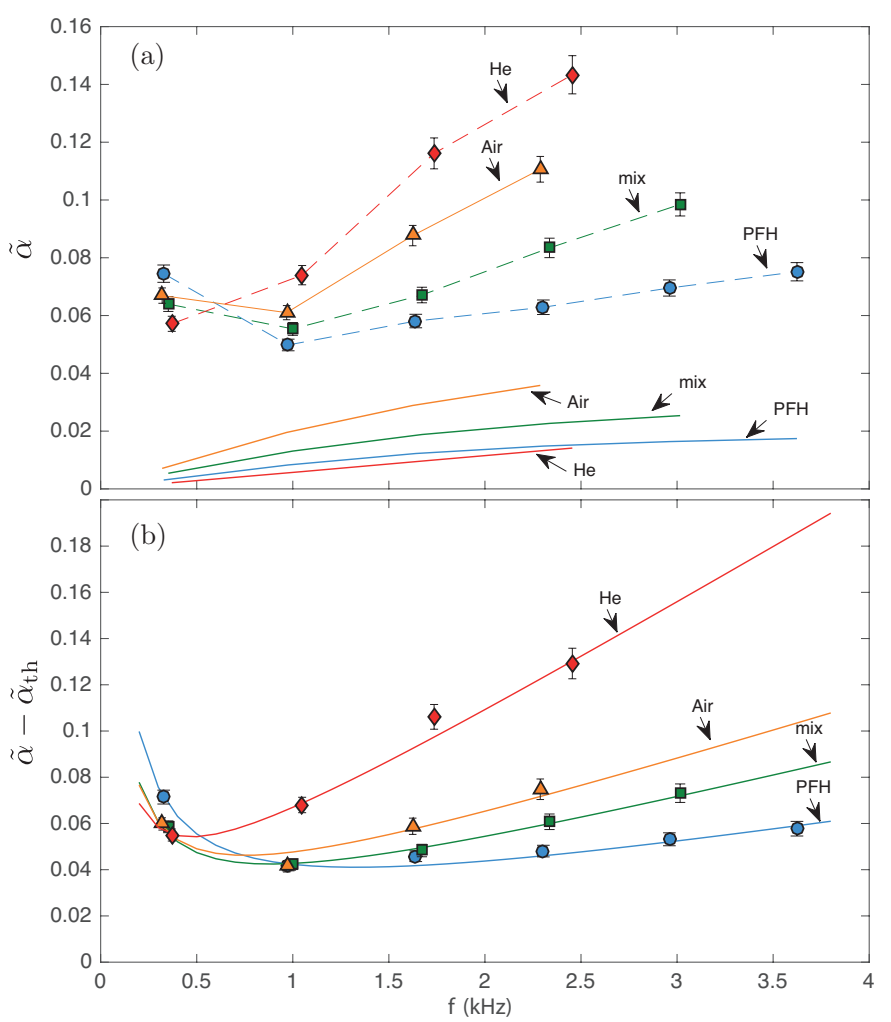

Fig. 8. (Color online) Reduced attenuation as a function of frequency for the four selected samples of Table 2. (a) Raw measurements (symbols) and thermal contributions predicted by the bubbly liquid approach (solid lines), taking account of the polydispersity of the foams. (b) When the thermal contribution is subtracted from the measurements (symbols), one can fit the data (solid lines) with Eq. (14), using two parameters: $\eta_{\mathrm{wall}}^{*}$ and $\eta_{\text {bub. }}$

\subsection{Viscous friction on the wall}

When a plane wave propagates in a tube, the displacement field needs to satisfy the boundary condition on the wall of the tube, which is done on a viscous layer, over which dissipation occurs. The attenuation associated with this phenomenon is given by Kirchhoff's law $[17,18]$

$$
\tilde{\alpha}_{\text {wall }}=\frac{\ell_{v}}{D}=\sqrt{\frac{2 \eta}{\Phi \rho_{\ell} D^{2}}} \omega^{-1 / 2},
$$

where $D$ is the diameter of the tube, $\ell_{v}$ the viscous length and $\eta$ the viscosity of the fluid in the tube. Note that we neglect the thermal contribution in Kirchhoff's law here. As the viscous layer thickness scales like $1 / \sqrt{\omega}$, the attenuation associated with this effect is larger at low frequencies, which makes it a good candidate for explaining our observations of Fig. 8. In usual conditions, this source of attenuation is negligible in impedance tubes, because they are designed with a diameter that is large enough compared to the viscous length in air $\left(\ell_{v} \simeq 70 \mu \mathrm{m}\right.$ for air at $1 \mathrm{kHz}$ ). For liquid foams, however, it appears that friction on the wall can become significant because the viscosity of the foam is high. Note that the existence of such a sheared layer close to a wall when a liquid foam is insonified was observed by Erpelding et al. [19].

We also confirmed the existence of this Kirchhoff attenuation in our samples by testing the role of tube diameter D. As shown in Appendix C, the same intrinsic attenuation was recovered for similar samples measured in two different tubes, provided that the wall attenuation was properly subtracted.

\subsection{Fitting}

We have identified three possible dissipation mechanisms involved in our experiment: two are intrinsic, with thermal and viscous origins, and one is caused by the experimental setup, the viscous friction on the wall. If we assume that thermal attenuation is known and given by the bubbly liquid approach, we are left with two viscous dissipation terms involving two unknown viscosities:

- viscosity $\eta_{\text {bub }}$ at the bubble level (appearing in Eq. 9), i.e. the viscosity experienced by one bubble of the foam during its oscillations,

- viscosity $\eta_{\text {wall }}$ on the wall (appearing in Eq. 12), which is the viscosity involved in the viscous layer to satisfy the boundary conditions on the tube.

Assuming that the reduced attenuation terms add up, as in Eq. (6), we thus have the following equation as a candidate for describing the experimental data of Fig. 8b:

$$
\begin{aligned}
\tilde{\alpha}-\tilde{\alpha}_{\mathrm{th}} & =\tilde{\alpha}_{\mathrm{wall}}+\tilde{\alpha}_{\mathrm{vi}} \\
& =\sqrt{\frac{2 \eta_{\mathrm{wall}}(\omega)}{\Phi \rho_{\ell} D^{2}}} \omega^{-1 / 2}+\frac{2 \eta_{\mathrm{bub}}(\omega)}{3 P_{0}} \omega .
\end{aligned}
$$

Note that the two unknown viscosities are a priori frequency dependent: $\eta_{\text {wall }}(\omega)$ and $\eta_{\text {bub }}(\omega)$. The next step is to determine this frequency dependence in order to have a fitting law for our $\tilde{\alpha}-\tilde{\alpha}_{\text {th }}$ vs $f$ plots. Among the choices already discussed in section 3.2 , two viscosities are frequency independent $\left(\eta_{\text {water }}\right.$ and $\left.\eta_{\text {channel }}\right)$ and one scales like $\omega^{-1 / 2}\left(\eta_{\text {foam }}\right)$. The high frequency behaviour 
of the plots shown in Fig. 8b suggest a linear law in frequency, hence a $\eta_{\text {bub }}$ that is frequency independent. On the other hand, trying to fit with a frequency-independent $\eta_{\text {wall leads to a } \omega^{-1 / 2}}$ law for $\tilde{\alpha}_{\text {wall }}$, which we found not to decrease fast enough with frequency to capture the behaviour depicted in Fig. 8b. With $\eta_{\text {wall }} \sim \omega^{-1 / 2}$, the low frequency part of the reduced attenuation scales like $\omega^{-3 / 4}$, which gave much better results for the fitting. It therefore suggests that $\eta_{\text {wall }}$ might correspond to $\eta_{\text {foam }}$. Assuming that $\eta_{\text {wall }}(\omega)=\eta_{\text {wall }}^{*} \sqrt{\omega^{*} / \omega}$, where $\omega^{*}=2 \pi \times$ $1 \mathrm{kHz}$, we thus obtain the following fitting law

$$
\tilde{\alpha}-\tilde{\alpha}_{\mathrm{th}}=\sqrt{\frac{2 \eta_{\mathrm{wall}}^{*} \sqrt{\omega^{*}}}{\Phi \rho_{\ell} D^{2}}} \omega^{-3 / 4}+\frac{2 \eta_{\mathrm{bub}}}{3 P_{0}} \omega,
$$

with the two fitting parameters $\eta_{\text {wall }}^{*}$ and $\eta_{\text {bub }}$. Solid lines in Fig. 8b show that satisfactory fittings are obtained with this law. The best fitting parameters we obtained for all the samples are displayed in Fig. 9. Error bars come from the $95 \%$ confidence bounds on the fit parameters.
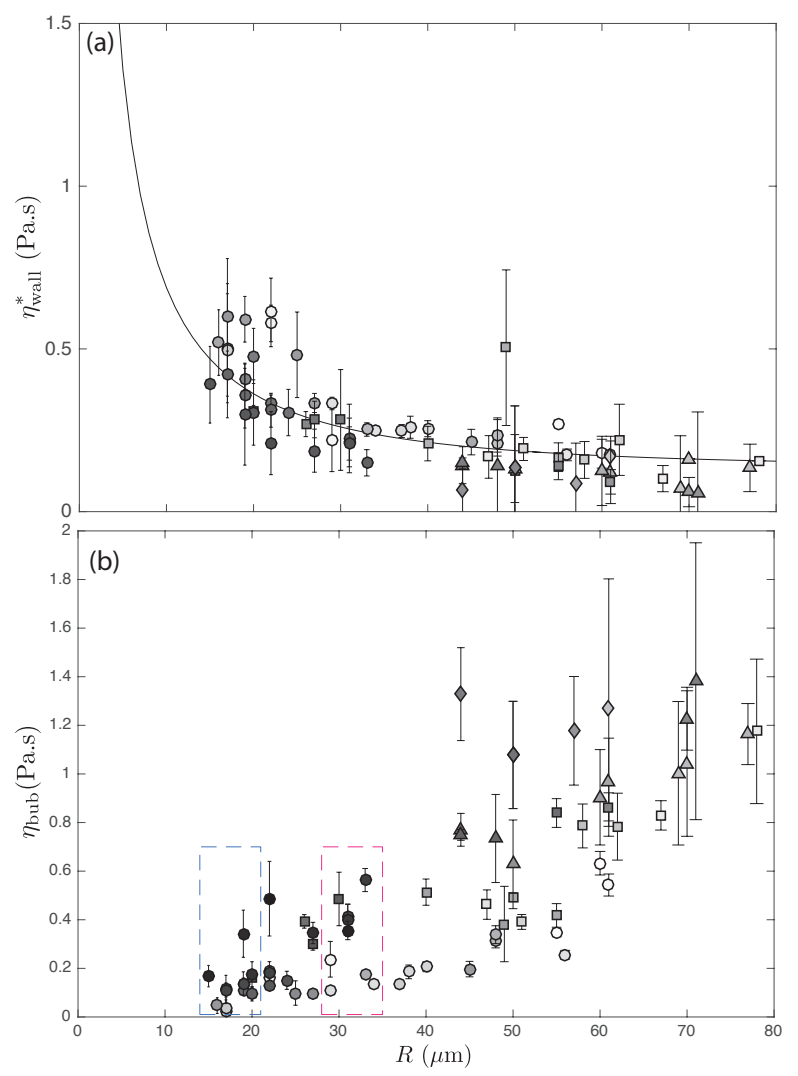

Fig. 9. Viscosities $\eta_{\text {wall }}^{*}$ (a) and $\eta_{\text {bub }}$ (b) as functions of $R$. Symbols code for the type of gas (see Table 1). The grey level of the symbols correspond to liquid fraction: the darker the symbols the wetter the foams (same as fig. 2). In (a) the continuous line indicates the $\eta_{\text {foam }}$ viscosity expected at $1 \mathrm{kHz}$, as given by phenomenological law (10), with no fitting parameter.

Both viscosities were found to mostly depend on the median radius $R$. Interestingly, values of $\eta_{\text {wall }}^{*}$ are close to prediction of Eq. (10), suggesting that the phenomenological law found by Costa et al. [16] with rheology measurements is still valid in the $\mathrm{kHz}$ frequency range, as already observed by Wintzenrieth et al for shaving foams [20].

As for $\eta_{\text {bub }}$, its values are two to three orders of magnitude higher than for pure water, and it increases with $R$. We shall explore its dependence on $R, \Phi$ and gas nature in further details in the next section.

\subsection{Gas and structure dependence of $\eta_{\text {bub }}$}

In addition to the clear dependence in $R$, a $\Phi$-dependence seems visible in Fig. 9b: darker symbols tend to correspond to higher values of viscosity than lighter ones, which would indicate that the viscosity is larger for wetter foam. To better appreciate this effect, we isolate two groups of samples of same gas composition $(\mathrm{PFH})$ and with median radius of the order of 20 (blue dashed rectangle) and $30 \mu \mathrm{m}$ (red dashed rectangle). The corresponding data points are displayed in Fig. 10, as function of liquid volume fraction $\Phi$. The values are scattered and no clear tendency can be extracted; $\eta_{\text {bub }}$ may be independent of or slightly increasing with $\Phi$. In any case, these measurements are not compatible with the Darcy model proposed by Goldfarb et al, which predicts a $1 / \Phi$ behaviour (see Eq. (11)). At this stage, it appears that none of the three candidates presented in section $3.2\left(\eta_{\text {water }}, \eta_{\text {channel }}\right.$ and $\left.\eta_{\text {foam }}\right)$ are compatible with our measurements of $\eta_{\text {bub }}$.

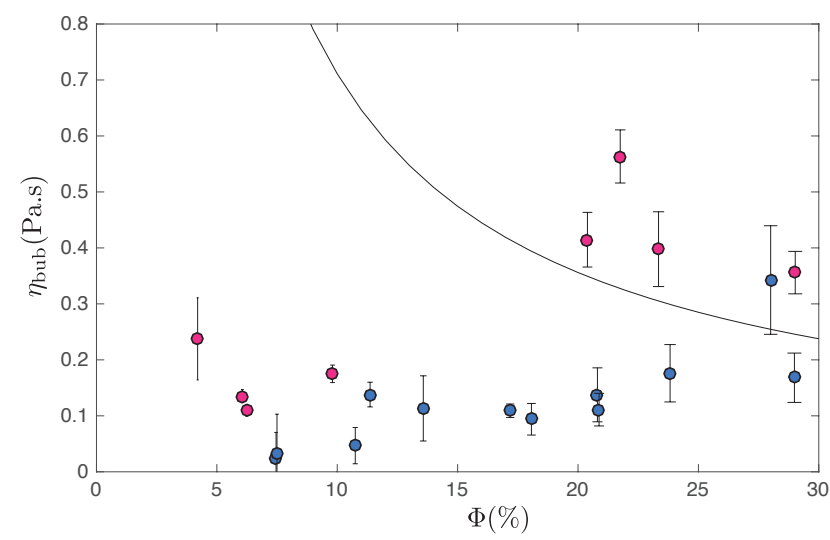

Fig. 10. Results of the fitting for $\eta_{\text {bub }}$ as a function of liquid volume fraction $\Phi$ for PFH foams with median radius of the order of $20 \mu \mathrm{m}$ (in blue) and $30 \mu \mathrm{m}$ (in red). The two sets of data points correspond to the blue and red dashed rectangles shown in Fig. 9. Solid line shows $\eta_{\text {channel }}$, the viscosity proposed by Goldfarb et al (see Eq. (11)).

We then turn to the effect of the nature of the gas on the acoustic attenuation. As Fig. 9a gives us confidence in identifying $\eta_{\text {wall }}$ with $\eta_{\text {foam }}$, we go a step further in our subtracting process and plot, in Fig. 11, $\tilde{\alpha}-\tilde{\alpha}_{\text {th }}-\tilde{\alpha}_{\text {wall }}$ (i.e. what we expect to correspond to $\tilde{\alpha}_{\mathrm{vi}}$ ), for the four samples that have similar structures and different gas contents (see Tab. 1). The linear behaviour, already visible in Fig. 8, is 
even clearer. Fitting linear laws through the data points then gives access to the values of $\eta_{\text {bub }}$ for the different gases. As shown in the inset of Fig. 11, the found values are compatible with a $1 / \sqrt{\rho_{g}}$ scaling law, suggesting that the lighter the gas of the foam, the larger the attenuation.

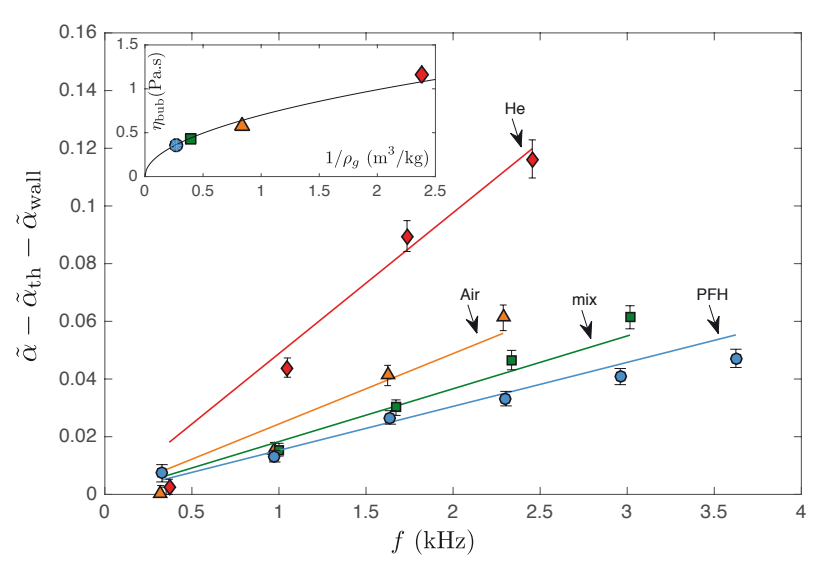

Fig. 11. Measured reduced attenuation without the thermal and the viscous friction on the wall contributions (markers) fitted by a linear law (continuous lines). Inset: $\eta_{\text {bub }}$ (extracted from the linear fit and using Eq.9) as a function of $1 / \rho_{g}$ (see Table 1).

To summarise the dependence of $\eta_{\text {bub }}$ on gas and bubble size, we plot in Fig. 12 the measured viscosities rescaled by $\sqrt{\rho_{g}}$ as a function of median radius $R$. As the effect of $\Phi$ has not been clearly identified, we limit ourselves to intermediate liquid volume fractions: $7<\Phi<15 \%$ in this figure. A reasonable collapse of the data points for different gas content is obtained, on a master curve that is compatible with a $R^{2}$ scaling.

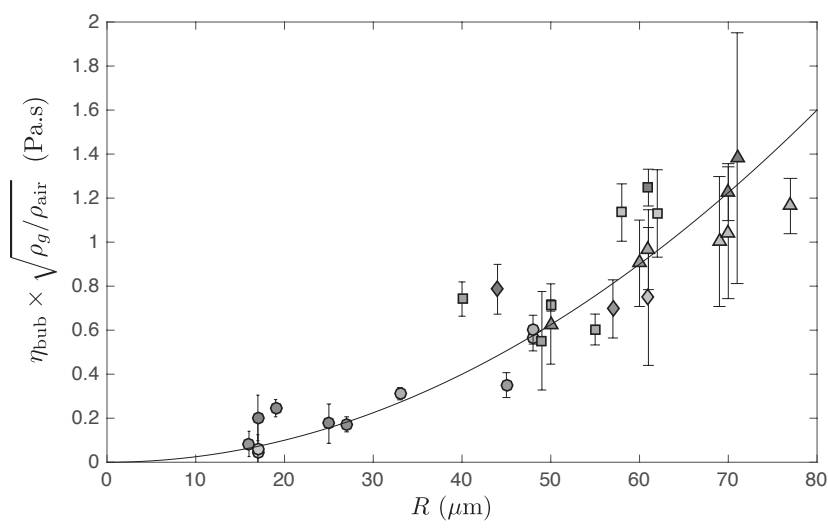

Fig. 12. Effective viscosity $\eta_{\text {bub }}$ as a function of $R$ for samples with liquid fraction between 7 and $15 \%$. Symbols code for the type of gas (see Table 1). Solid line corresponds to $(R / 50 \mu \mathrm{m})^{2} \times 0.6$ Pa.s.

\section{Conclusion}

In this article, we presented experimental results on the attenuation of acoustic waves in liquid foams of well controlled structure and composition.

By systematically analysing the influence of the frequency $(0.4<f<4 \mathrm{kHz})$, the median radius of the bubbles $(R)$, the liquid volume fraction $(\Phi)$ and the nature of the gas, we managed to identify three contributions to the attenuation.

An attenuation $\tilde{\alpha}_{\text {wall }}$ that is due to our setup (an impedance tube) and is found to be well accounted by viscous friction on the wall with the macroscopic viscosity of the foam, which depends on the bubble size and follows the law established by rheology measurements at lower frequencies.

An intrinsic attenuation $\tilde{\alpha}_{\text {th }}$ that is due to thermal losses during the oscillation of the bubbles when the acoustic waves propagates. We assumed that this attenuation was the same as in bubbly liquids, which is supported by (1) our measurements of sound velocities compatible with an isothermal Wood's law, (2) our calculations of the effect of thin films on the thermal behaviour of the bubbles (Appendix B).

An other intrinsic attenuation $\tilde{\alpha}_{\mathrm{vi}}$ that is due, in our modelling, to the flow the bubbles generate during their oscillation. This last term is intriguing and, contrary to the two previous ones, we cannot associate it with any clear dissipation mechanism. According to our measurements, a phenomenological law for this attenuation would be

$$
\tilde{\alpha}_{\mathrm{vi}} \simeq 0.025 \frac{\omega}{\omega^{*}}\left(\frac{R}{R^{*}}\right)^{2}\left(\frac{\Phi}{\Phi^{*}}\right)^{a} \sqrt{\frac{\rho_{\mathrm{air}}}{\rho_{g}}},
$$

with reference radius $R^{*}=50 \mu \mathrm{m}$, frequency $\omega^{*}=2 \pi \times$ $1 \mathrm{kHz}$, and volume fraction $\Phi^{*}=10 \%$, and with an exponent $a \geq 0$. Interestingly, we recover the $\omega R^{2}$ scaling law that had been associated with thermal losses in previous studies. However, we insist, thermal attenuation cannot alone explain our observations: (1) it predicts too small values of attenuation (see Fig. 8a) and (2) modifications of the current model would tend to lead to even lower values, not larger, (3) helium foams would be expected to attenuate the least, while we found the opposite.

As a final remark, we note that the observed dependence of $\tilde{\alpha}_{\mathrm{vi}}$ to the density of the gas suggests that its origin might actually be quite different from the mechanism we invoke for establishing it. Further experimental and theoretical works will be necessary for elucidating the origin of this acoustic attenuation.

Support for French Agence National de la Recherche (project SAMOUSSE, ANR-11-BS09-001) is gratefully acknowledged. The authors thank Wiebke Drenckhan for fruitful discussions.

\section{A Thermal properties of gas mixtures}

Thermal properties of a gas mixture were estimated by calculating averaged values of the thermal conductivity 
$(\lambda)$ and the heat capacity $\left(C_{p}\right)$ :

$$
\begin{aligned}
\lambda & =x \lambda_{1}+(1-x) \lambda_{2}, \\
C_{p} & =x C_{p, 1}+(1-x) C_{p, 2},
\end{aligned}
$$

where $x$ is the molar fraction of gas 1 in the mixture. These linear laws are only approximations [21], but they provide good orders of magnitudes, and we checked that the final results in terms of thermal attenuation were not too sensitive to the exact values of the parameters. With equations (16), we then estimated $\gamma$ and $D_{\text {th }}$ with the following formulas:

$$
\begin{aligned}
\gamma & =\frac{C_{p}}{C_{p}-R}, \\
D_{\mathrm{th}} & =\frac{\lambda R T}{P_{0} C_{p}},
\end{aligned}
$$

where $R \simeq 8.31 \mathrm{~J} / \mathrm{K} / \mathrm{mol}$ and $T$ is the temperature.

\section{B Thermal exchanges through thin films}

In this appendix, we propose to investigate the role of thin films in the thermal exchanges between gas bubbles. Indeed, the bubbly liquid approximation assumes that the temperature of the liquid around each bubble remain constant during the oscillations. This assumption is questionable when only a thin layer of liquid separates the bubbles.

Note that Goldfarb and coworkers calculated the effect of a finite amount of liquid on the thermal behavior of a bubble [12]. However, they disregarded the role of the films, because they considered a uniform layer of liquid around each bubble, which is not realistic for foams. Furthermore, their condition of zero heat flux for the outer part of the liquid layer is questionable, as it implies that no thermal exchange can exist between neighbouring bubbles. For simplicity, we consider a one-dimension problem,

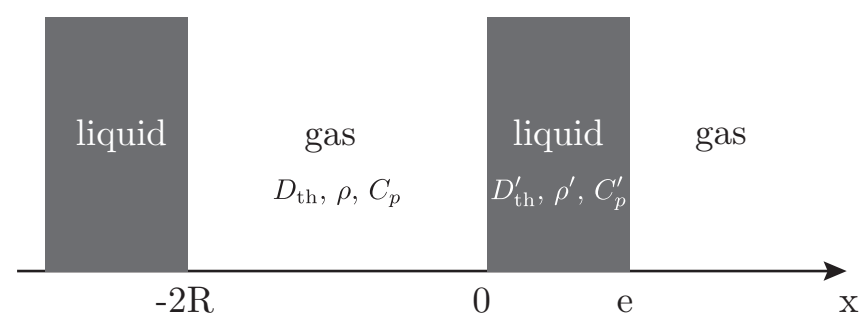

Fig. 13. One-dimension study of thermal exchange between air bubbles separated by thin films. We calculate the temperature profile and the volume variation of the gas layer when an extra pressure $p \exp [-\mathrm{i} \omega t]$ is applied.

as schematized in figure 13: gas layers of length $2 R$ are separated by liquid walls of thickness $e$. We want to calculate the volume variation of the gas layer when a pressure variation $p \exp [-\mathrm{i} \omega t]$ is applied. The main question is to determine whether this volume variation will be isothermal or adiabatic, depending on the heat exchange between the gas and the liquid. Heat equation applies in each layer:

$$
\frac{\partial}{\partial t} T(x, t)-D_{\mathrm{th}} \Delta T(x, t)=\frac{1}{\rho C_{p}} \frac{\partial P}{\partial t} .
$$

We look for solutions of the form

$$
T(x, t)=T_{0}+T_{1}(x) \exp [-\mathrm{i} \omega t]
$$

( $T_{0}$ being the temperature at equilibrium), with temperature profiles

$$
\begin{aligned}
T_{1}(x) & =\frac{p}{\rho C_{p}}\left[1+A \cos \left(k_{\mathrm{th}} x\right)+B \sin \left(k_{\mathrm{th}} x\right)\right], \\
\text { for }-2 R<x & <0 \text { and } \\
T_{1}(x) & =\frac{p}{\rho^{\prime} C_{p}^{\prime}}\left[1+A^{\prime} \cos \left(k_{\mathrm{th}}^{\prime} x\right)+B^{\prime} \sin \left(k_{\mathrm{th}}^{\prime} x\right)\right]
\end{aligned}
$$

for $0<x<e$, where $k_{\mathrm{th}}=\left(\mathrm{i} \omega / D_{\mathrm{th}}\right)^{1 / 2}$. Constants $A, B$, $A^{\prime}$, and $B^{\prime}$ are determined by imposing the continuity of temperature and heat flux both in $x=0$ and between $x=$ $-2 R$ and $x=+e$ (periodic conditions). Then, knowing the profile of temperature in the gas, one can determine the small volume variation $(\mathrm{d} V)$ in between $x$ and $x+$ $\mathrm{d} x$, and integrate to calculate the total volume variation, which in turn leads to the compressibility $\chi=1 /\left(\kappa P_{0}\right)=$ $-(1 / V) \partial V / \partial P$.

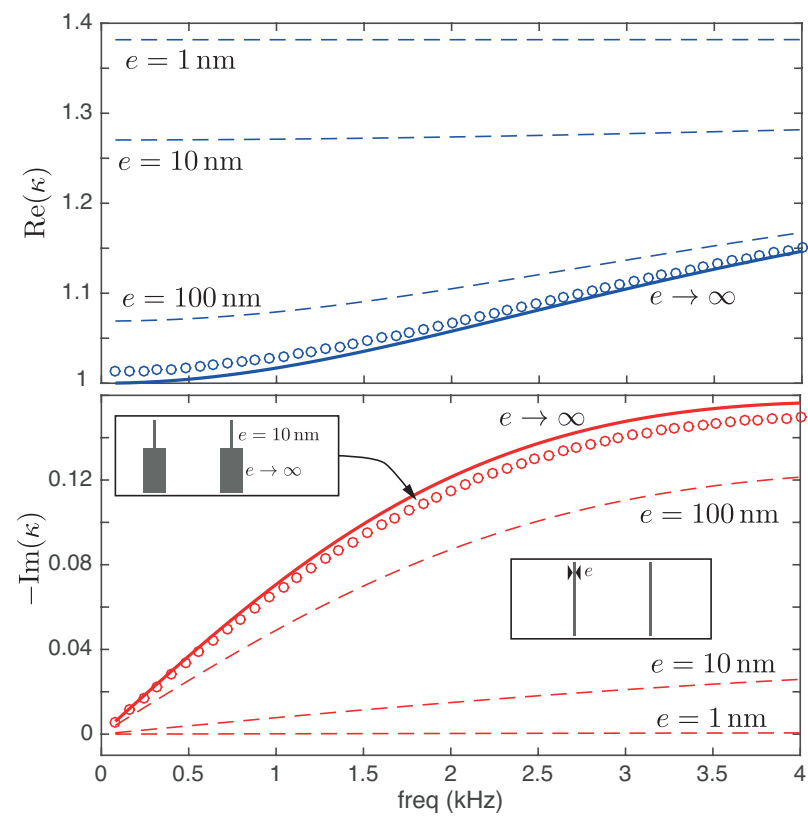

Fig. 14. Real (top) and imaginary (bottom) part of the polytropic exponent $\kappa$ as functions of frequency, for the onedimension case depicted in Fig. 13. We consider air layers of $2 R=100 \mu \mathrm{m}$, and water walls of different thicknesses $e$. Thick lines show the results for an infinite amount of water $(e \rightarrow \infty)$. Circles are for the case of two walls in parallel: $20 \%$ of the surface being a $10 \mathrm{~nm}$ film, and $80 \%$ an "infinite" wall. 
Figure 14 shows results of this calculation for air layers of $2 R=100 \mu \mathrm{m}$ separated by water films of different thicknesses $e$. As expected, for large thicknesses, $\kappa$ tends toward the "bubbly liquid" behaviour (slightly different here because we consider a $1 \mathrm{D}$ problem). In particular, $\operatorname{Re}(\kappa)$ remains close to 1 . On the other hand, for films as thin as $1 \mathrm{~nm}$, the adiabatic behaviour is recovered $(\operatorname{Re}(\kappa) \simeq 1.4)$ and the thermal attenuation is significantly reduced. We thus obtain the intuitive result that if films are too thin, they do not thermally separate the air layers. From our simple model, "too thin" here roughly means less than $1 \mu \mathrm{m}$. In liquid foams, typical films thickness is of the order of tens of nanometers, which means that clear deviation from the bubbly liquid case could be expected. However, not the whole surface of bubbles is covered by films; there are also liquid channels whose thermal inertia is much higher. We propose to account for the presence of both films and channels by considering two walls in parallel, as depicted in the inset of Fig. 14, and calculate an average polytropic exponent given by $1 / \kappa=x / \kappa_{\text {film }}+(1-x) / \kappa_{\text {channel }}$, where $x$ is the surface ratio covered by the films around a bubble. For foams of liquid volume fraction $10 \%$, this ratio is of the order of $x=0.2[22]$. As shown by the circles in Fig. 14, with this model even $10 \mathrm{~nm}$ films bring a negligible deviation from the pure bubbly liquid case.

\section{Viscous friction on the wall}

The analysis of our measurements suggests that part of the losses for the acoustic waves is due to friction on the wall of the tube, with the following reduced attenuation (see section 4.3):

$$
\tilde{\alpha}_{\text {wall }}=\frac{\omega^{-3 / 4}}{D} \sqrt{\frac{2 A \sqrt{1+(B / R)^{2}}}{\Phi \rho_{\ell}}},
$$

with $A=10.3$ Pa.s ${ }^{1 / 2}$ and $B=52 \mu \mathrm{m}$. This relationship can be further tested by comparing measurements on similar foams in tubes of different diameters. Table 3 shows the results of two tests, comparing measurements for $D=100 \mathrm{~mm}$ and $D=29 \mathrm{~mm}$. One can observe that measured reduced attenuations $\tilde{\alpha}$ are larger in the narrower tube. By subtracting the $\tilde{\alpha}_{\text {wall }}$ predicted by Eq. $(22)$ from the measured $\tilde{\alpha}$, we recover the same intrinsic attenuations. (Appendix A reports how the thermal )

Table 3. Acoustic attenuation at $0.5 \mathrm{kHz}$ for $\mathrm{PFH}$ liquid foams measured in two impedance tubes of different diameters.

\begin{tabular}{|c|c|c|c|c|c|}
\hline $\begin{array}{c}D \\
(\mathrm{~mm})\end{array}$ & $\begin{array}{c}R \\
(\mu \mathrm{m})\end{array}$ & $\begin{array}{c}\mathrm{PI} \\
(\%)\end{array}$ & $\begin{array}{c}\Phi \\
(\%)\end{array}$ & $\tilde{\alpha}$ & $\tilde{\alpha}-\tilde{\alpha}_{\text {wall }}$ \\
\hline 100 & 32 & 40 & 10 & 0.22 & 0.13 \\
29 & 33 & 24 & 10.5 & 0.51 & 0.13 \\
\hline 100 & 20 & 35 & 16 & 0.19 & 0.09 \\
29 & 22 & 35 & 19 & 0.48 & 0.09 \\
\hline
\end{tabular}

\section{References}

1. Juliette Pierre, Benjamin Dollet, and Valentin Leroy. Resonant acoustic propagation and negative density in liquid foams. Physical Review Letters, 112(148307), 2014.

2. Albert Beaumont Wood. A text book of sound. G. Bell and Sons, London, 1941.

3. KB Kann. Sound waves in foams. Colloids and surfaces a: physicochemical and engineering aspects, 263(1):315-319, 2005.

4. Richard Raspet and SK Griffiths. The reduction of blast noise with aqueous foam. The Journal of the Acoustical Society of America, 74(6):1757-1763, 1983.

5. Nicolás Mujica and Stéphan Fauve. Sound velocity and absorption in a coarsening foam. Physical Review E, 66(2):021404, 2002.

6. Martin Monloubou, Arnaud Saint-Jalmes, Benjamin Dollet, and Isabelle Cantat. Influence of bubble size and thermal dissipation on compressive wave attenuation in liquid foams. EPL (Europhysics Letters), 112(3):34001, 2015.

7. I Goldfarb, Z Orenbakh, I Shreiber, and F Vafina. Sound and weak shock wave propagation in gas-liquid foams. Shock Waves, 7(2):77-88, 1997.

8. Juliette Pierre, Reine-Marie Guillermic, Florence Elias, and Valentin Leroy. Acoustic characterisation of liquid foams with an impedance tube. European Physical Journal E, 36(113), 2013.

9. II Goldfarb, ZM Orenbakh, GA Shushkov, IR Shreiber, and FI Vafina. Investigation of sound waves propagation peculiarities in gas-liquid foam. Le Journal de Physique IV, 2(C1):C1-891, 1992.

10. T. Gaillard, M. Roché, C. Honorez, M. Jumeau, A. Balan, C. Jedrzejczyk, and W. Drenckhan. Controlled foam generation using cyclic diphasic flows through a constriction. International Journal of Multiphase Flow, 2017.

11. Thibaut Gaillard, Clément Honorez, Maxime Jumeau, Florence Elias, and Wiebke Drenckhan. A simple technique for the automation of bubble size measurements. Colloids and Surfaces A: Physicochemical and Engineering Aspects, 473:68-74, 2015.

12. II Goldfarb, IR Schreiber, and FI Vafina. Heat transfer effect on sound propagation in foam. The Journal of the Acoustical Society of America, 92(5):2756-2769, 1992.

13. Imen Ben Salem, Reine-Marie Guillermic, Caitlin Sample, Valentin Leroy, Arnaud Saint-Jalmes, and Benjamin Dollet. Propagation of ultrasound in aqueous foams: bubble size dependence and resonance effects. Soft Matter, 9(4):1194-1202, 2013.

14. Andrea Prosperetti. Nonlinear oscillations of gas bubbles in liquids: steady-state solutions. The Journal of the Acoustical Society of America, 56(3):878-885, 1974.

15. Isabelle Cantat, Sylvie Cohen-Addad, Florence Elias, François Graner, Reinhard Höhler, Olivier Pitois, Florence Rouyer, and Arnaud Saint-Jalmes. Foams: structure and dynamics, 2013.

16. Séverine Costa, Reinhard Höhler, and Sylvie CohenAddad. The coupling between foam viscoelasticity and interfacial rheology. Soft Matter, 9(4):1100-1112, 2013.

17. Gustav Kirchhoff. Ueber den einfluss der wärmeleitung in einem gase auf die schallbewegung. Annalen der Physik, 210(6):177-193, 1868.

18. DE Weston. The theory of the propagation of plane sound waves in tubes. Proceedings of the Physical Society. Section B, 66(8):695, 1953. 
19. Marion Erpelding, Reine-Marie Guillermic, Benjamin Dollet, Arnaud Saint-Jalmes, and Jérôme Crassous. Investigating acoustic-induced deformations in a foam using multiple light scattering. Physical Review E, 82(2):021409, 2010.

20. Frédéric Wintzenrieth, Sylvie Cohen-Addad, Marie Le Merrer, and Reinhard Höhler. Laser-speckle-visibility acoustic spectroscopy in soft turbid media. Physical Review E, 89(1):012308, 2014.

21. Alexander L Lindsay and LeRoy A Bromley. Thermal conductivity of gas mixtures. Industrial \& Engineering Chemistry, 42(8):1508-1511, 1950.

22. HM Princen. Rheology of foams and highly concentrated emulsions. ii. experimental study of the yield stress and wall effects for concentrated oil-in-water emulsions. Journal of colloid and interface science, 105(1):150-171, 1985. 